\title{
MicroRNA expression profiles identify biomarkers for predicting the response to chemoradiotherapy in rectal cancer
}

\author{
BINBIN DU ${ }^{1}$, XIAOYING WANG ${ }^{1}$, DEWANG WU ${ }^{1}$, TAO WANG $^{1}$, XIONGFEI YANG ${ }^{1}$, \\ JIANKAI WANG ${ }^{2}$, XINLONG SHI $^{1}$, LINGJUAN CHEN ${ }^{1}$ and WEISHENG ZHANG ${ }^{1}$
}

Departments of ${ }^{1}$ Anorectal Surgery and ${ }^{2}$ Radiotherapy, Gansu Provincial Hospital, Lanzhou, Gansu 730000, P.R. China

Received July 11, 2017; Accepted November 2, 2017

DOI: $10.3892 / \mathrm{mmr} .2018 .9215$

\begin{abstract}
Neoadjuvant chemoradiotherapy (nCRT) following surgery significantly improves the survival rate of patients with rectal cancer. However, nCRT is associated with significant adverse symptoms and high medical costs. Therefore, it is important to investigate potential biomarkers for the prediction of the response to nCRT in patients with rectal cancer. The present study identified candidate biomarkers for predicting a complete response (CR) to nCRT in patients with rectal cancer and investigated the associated mechanisms. Microarray data (accession no. GSE29298) was downloaded from the Gene Expression Omnibus database. Differentially expressed microRNAs (miRNAs/miR) were screened between the pathological CR (pCR) group and no pCR (incomplete response) group. miRNA target genes were predicted using the miRWalk 2.0 online tool and subjected to Kyoto Encyclopedia of Genes and Genomes pathway enrichment analysis. Furthermore, a miRNA co-regulatory network was constructed and disease-associated genes were predicted. The results demonstrated that a total of 36 upregulated and 5 downregulated miRNAs were identified between the two groups. Among these differentially expressed miRNAs, miR-548c-5p, miR-548d-5p and miR-663a were significantly associated with a CR to nCRT. The co-regulatory network and pathway analysis indicated that miR-548c-5p and miR-548d-5p may function together through stem cell pluripotency and ubiquitin-mediated proteolysis signaling pathways. Furthermore, the prediction of disease-associated genes demonstrated that $\mathrm{miR}-548 \mathrm{c}-5 \mathrm{p} / \mathrm{miR}-548 \mathrm{~d}-5 \mathrm{p}$ and $\mathrm{miR}-663 \mathrm{a}$ may regulate genes associated with rectal cancer, including mutated in colorectal cancers $(M C C)$ and adenomatous polyposis coli $(A P C)$, and colorectal neoplasms, including interleukin-6 signal transducer (IL6ST), cell cycle checkpoint kinase 2
\end{abstract}

Correspondence to: Dr Weisheng Zhang, Department of Anorectal Surgery, Gansu Provincial Hospital, 204 Donggang West Road, Chengguan, Lanzhou, Gansu 730000, P.R. China

E-mail: shang9368231908@163.com

Key words: rectal cancer, microRNA, neoadjuvant chemoradiotherapy, pathological response, prediction
(CHEK2), marker of proliferation Ki-67 (MKI67), cadherin $7(C D H 7)$, calreticulin $(C A L R)$ and transforming growth factor $\beta 1$ (TGFB1). Therefore, miR-548c-5p, miR-548d-5p and miR-663a are promising candidate biomarkers for predicting a CR to nCRT. miR-548c-5p/miR-548d-5p may be associated with a CR by regulating IL6ST, CHEK2, MKI67 and MCC. In addition, it may function through the pluripotency of stem cells and ubiquitin-mediated proteolysis signaling pathways. miR-663a may be associated with a CR to nCRT by targeting $C D H 7, C A L R, A P C$ and TGF $\beta 1$. Thus, the miRNA biomarkers investigated in the present study may represent novel therapeutic targets for the prediction and eventual improvement of the response to nCRT in patients with rectal cancer.

\section{Introduction}

Colorectal cancer (CRC) is the third most common type of malignant tumor in the digestive tract and poses a substantial threat to human health worldwide (1). In addition, CRC is the third highest contributor to cancer-associated mortality worldwide, with $\sim 26,580$ and 24,790 mortalities annually for males and females, respectively (2). It is reported that $20 \%$ of all patients with CRC are diagnosed with rectal cancer, while $\sim 45 \%$ of patients with rectal cancer are further diagnosed with locally advanced rectal cancer (LARC) $(3,4)$.

Local recurrence is common in patients with LARC. Neoadjuvant chemoradiotherapy (nCRT) may prevent local recurrence and improve overall survival, and has therefore emerged as the standard treatment for patients with LARC, followed by surgical resection (5). Overall, following nCRT, $\sim 15-20 \%$ of patients with LARC demonstrate a complete response (CR), with complete tumor eradication (6-8) while $20 \%$ of patients exhibit a poor response to nCRT (9-11). Patients with a CR to nCRT subsequently undergo a simple watch-and-wait procedure or endoscopic resection (12). Patients with a poor response to nCRT are associated with substantial adverse effects and high medical costs (13). Thus, identifying valid predictive biomarkers is of high importance.

MicroRNAs (miRNAs/miR) have been proposed as potential biomarkers for predicting the response to treatment and prognosis in rectal cancers. The expression of miR-143 and miR-145 is reported to be significantly upregulated in rectal tumor tissues following treatment, and miR-145 expression demonstrated a close association with tumor regression 
in patients with rectal cancer (14). Additionally, the distinct expression signatures of miR-16, miR-590-5p and miR-153 in pretreated rectal cancer tissue may predict the response to treatment, miR-519c-3p and miR-561 accurately predict a good or poor response to nCRT in patients with rectal cancer (15). Furthermore, miR-21-5p was demonstrated to be overexpressed in patients with CR (16). However, the role of these biomarkers has not been fully clarified.

Della Vittoria Scarpati et al (17), reported a set of 13 miRNAs with specific signatures that were associated with a pathological CR (pCR) to nCRT based on microarray data (accession no. GSE29298). However, the mechanisms of the biomarkers remain unclear. Therefore, the present study downloaded this data from the Gene Expression Omnibus (GEO) database. Based on the miRNA expression profile, differentially expressed miRNAs were identified between pCR and no pCR (incomplete response) groups, and their target genes were predicted, followed by Kyoto Encyclopedia of Genes and Genomes (KEGG) pathway enrichment analysis, miRNA co-regulatory network construction and disease-associated gene analysis. The aim of the current study was to investigate specific miRNA signatures as potential biomarkers for predicting a CR to $\mathrm{nCRT}$ in rectal cancer and to determine the potential mechanisms of the miRNAs.

\section{Materials and methods}

Data acquisition. The miRNA expression profile of GSE29298 (17) was downloaded from the GEO (http://www.ncbi.nlm.nih.gov/geo/), which was sequenced on an Agilent-021827 Human miRNA Microarray platform (Agilent Technologies, Inc., Santa Clara, CA, USA). The miRNA expression profile was analyzed by microarray using fresh frozen biopsies. A total of 38 patients with LARC (cT3-4/N+) were treated with capecitabine-oxaliplatin and pelvic conformal radiotherapy (45cGy) followed by surgery (after 6-8 weeks). Pathological responses were scored according to the tumor regression grade (TRG), as described by Mandard (9). The number of patients with TRG1, TRG2, TRG3 and TRG4 was 9, 16, 10 and 3, respectively. TRG1 indicates pCR, which represents complete tumor regression, while TRG $>1$ represents an incomplete response (no pCR) with residual tumor cells. Patients were divided into two groups: pCR group (TRG1, n=9) and no pCR group (TRG >1, n=29).

Data preprocessing. Microarray data were preprocessed using the Bioconductor package (version 3.5) Limma (version 3.28.21) (18), which involved background correction, normalization and expression calculation. The miRNA ID was transformed from the probe ID according to the probe annotation file. The most recent human miRNA annotation file was downloaded from the miRBase Database $(19,20)$ to obtain the miRNA name from the miRNA ID.

Identification of differentially expressed miRNAs. Differentially expressed miRNAs in the pCR group compared with the no pCR group were identified by non-paired Student's $\mathrm{t}$ test analysis and linear models using the Bioconductor package Limma. $\mathrm{P}<0.05$ andllog $_{2}$ fold change $(\mathrm{FC}) \mid \geq 0.58$ were set as the cutoff criteria for differentially expressed miRNAs.
Prediction of target genes for differentially expressed miRNAs. Target genes regulated by the differentially expressed miRNAs were predicted by 'validated target module' using the miRWalk 2.0 online tool (http://zmf.umm.uni-heidelberg .de/apps/zmf/mirwalk2) (21).

KEGG pathway enrichment analysis. KEGG pathways enriched in miRNA target genes were analyzed using the clusterProfiler (version 3.3.1) of R package (version 3.3.2) (22). Pathways involving miRNAs were determined. The P-value was calculated using a hypergeometric distribution statistical approach based on the KEGG.db annotation package (version 3.2.3) (23). The cutoff value for a significant pathway was set at $\mathrm{P}<0.05$.

miRNA co-regulatory network. According to the association between miRNAs and target genes, miRNA-miRNA pairs that regulate the same target genes were screened and an miRNA co-regulatory network was constructed by Cytoscape software (version 3.2.0) (24).

Disease-associated gene analysis. Marker or therapeutic genes associated with colorectal neoplasms were downloaded from the Comparative Toxicogenomics Database (25). Subsequently, the regulatory network between differentially expressed miRNAs and disease-associated genes was constructed by Cytoscape software (version 3.2.0) (24).

\section{Results}

Differentially expressed miRNAs. Following preprocessing, 835 miRNAs were identified and subjected to non-paired Student's t test analysis. According to $\mathrm{P}<0.05$ and $\log _{2} \mathrm{FCl}$ $\geq 0.58$, a total of 41 miRNAs were differentially expressed in the pCR group compared with the no pCR group, including 36 upregulated and 5 downregulated miRNAs. The heat map demonstrated that the two groups were distinguished by the expression profiles of 41 differentially expressed miRNAs (Fig. 1).

Target genes regulated by differentially expressed miRNAs. As indicated in Table I, 3,989 target genes were predicted to be regulated by 41 differentially expressed miRNAs. The results revealed that a single miRNA regulates multiple genes. miR-548c-5p, miR-548d-5p and miR-663a were demonstrated to regulate the greatest number of genes, and were reported to regulate 274,277 and 127 genes, respectively.

Significant KEGG pathways of differentially expressed miRNAs. Significant pathways involving the top 11 differentially expressed miRNAs are illustrated in Fig. 2. For miR-548c-5p and miR-548d-5p, the significantly enriched pathways included 'signaling pathways regulating pluripotency of stem cells' and 'ubiquitin-mediated proteolysis'.

miRNAs and their co-regulatory target genes. A co-regulatory network with 41 nodes and 596 edges was constructed, as demonstrated in Fig. 3. The results indicated that the miR-202-3p/let-7e-5p pairing is responsible for the co-regulation 156 genes, while the miR-548c-5p/miR-548d-5p complex 
Table I. Target genes regulated by microRNAs in pathological complete response group compared with the pathological incomplete response group.

A, Upregulated miRNAs

miRNA

hsa-miR-1183

hsa-miR-1224-5p

hsa-miR-1246

hsa-miR-125a-3p

hsa-miR-1268a

hsa-miR-134-5p

hsa-miR-1471

hsa-miR-188-5p

hsa-miR-483-5p

hsa-miR-548c-5p

hsa-miR-548d-5p

hsa-miR-575

hsa-miR-622

hsa-miR-630

hsa-miR-659-3p

hsa-miR-663a

hsa-miR-671-5p

hsa-miR-765

hsa-miR-1182

hsa-miR-1207-5p

hsa-miR-1225-5p

hsa-miR-1226-5p

hsa-miR-1275

hsa-miR-1299

hsa-miR-149-3p

hsa-miR-150-3p

hsa-miR-1909-5p

hsa-miR-1914-3p

hsa-miR-202-3p

hsa-miR-370-3p

hsa-miR-371a-5p

hsa-miR-494-3p

hsa-miR-557

hsa-miR-584-5p

hsa-miR-601

hsa-miR-623

B, Downregulated miRNAs

miRNA

Target gene count

hsa-let-7e-5p

711

hsa-miR-1260a

170

hsa-miR-192-3p

149

hsa-miR-26a-5p

541

hsa-miR-30b-5p

477

108

84

52

292

49

95

3

136

145

274

277

141

118

83

68

127

164

355

82

223

49

78

155

84

733

44

50

117

253

111

324

154

207

79

18

262

miR/miRNA, microRNA. was demonstrated to co-regulate 183 genes, including interleukin (IL)-6 signal transducer (IL6ST), cell cycle checkpoint kinase 2 (CHEK2), mutated in colorectal cancers $(M C C)$, marker of proliferation Ki-67 (MKI6), damage-specific DNA binding protein 1 and actinin $\alpha 4$.

Rectal cancer-associated genes. A total 33 differentially expressed miRNAs associated with the regulation of rectal cancer-associated genes were analyzed. The regulatory network between 33 miRNAs and 80 marker or therapeutic genes associated with colorectal neoplasms was constructed (Fig. 4). Four genes, including cyclin-dependent kinase inhibitor 1B (CDKNIB), adenomatous polyposis coli $(A P C)$, $M C C$ and interferon $\beta 1$ (IFNBI), were demonstrated to be associated with rectal cancer. The following seven miRNAs were identified as regulators of the four rectal cancer-associated genes: $\mathrm{miR}-548 \mathrm{c}-5 \mathrm{p} / \mathrm{miR}-548 \mathrm{~d}-5 \mathrm{p}(M C C)$, miR-663a $(A P C)$, miR-149-3p (CDKN1B), miR-1909-5p (CDKN1B), miR-494-3p (MCC) and miR-26a-5p (IFNB1). Specifically, the $\mathrm{miR}-548 \mathrm{c}-5 \mathrm{p} / \mathrm{miR}-548 \mathrm{~d}-5 \mathrm{p}$ complex was reported to co-regulate IL6ST, CHEK2, MCC and MKI67, and miR-663a was demonstrated to regulate cadherin $7(\mathrm{CDH})$, calreticulin $(C A L R), A P C$ and transforming growth factor (TGF) $\beta 1$ (TGFB1).

\section{Discussion}

It was previously reported that nCRT improves the outcome of patients with rectal cancer; however, not all patients benefit from this treatment (15). Thus, identifying predictive molecular biomarkers is of clinical importance (16). The present study aimed to identify specific miRNAs as predictive biomarkers based on their expression profiles. Furthermore, the potential underlying mechanisms of the biomarkers involved in the response to nCRT were investigated. In the current study, 36 upregulated and 5 downregulated miRNAs were detected between pCR and no pCR (incomplete response) groups.

KEGG pathway analysis demonstrated that the differentially expressed miR-134-5p and miR-630 were significantly associated with the 'HIF-1 signaling pathway'. Hypoxia-inducible factor (HIF)-1 is a transcription factor that affects gene expression by regulating oxygen delivery and deprivation (26). In addition, the activation of the HIF pathway is reported to contribute to tumor growth. Clinical evidence demonstrated that HIF-1 was overexpressed in patients with a poor prognosis in various cancer types (27). In the present study, miR-134-5p and miR-630 were demonstrated to be significantly upregulated in patients that exhibited a CR to nCRT. The expression of miRNA is negatively associated with the expression of its target genes. Thus, the target genes involved in the HIF-1 signaling pathway were significantly downregulated in CR cases, which may inhibit the HIF-1 signaling pathway and contribute to tumor regression. These results indicated that the bioinformatic findings were promising in the present study. Additionally, three miRNAs among the top 11 miRNAs, including miR-548c-5p, miR-548d-5p and miR-663a, were demonstrated to regulate genes associated with colorectal neoplasms.

It has been previously reported that miR-548c-5p inhibited the proliferation, migration and invasion, and promoted 


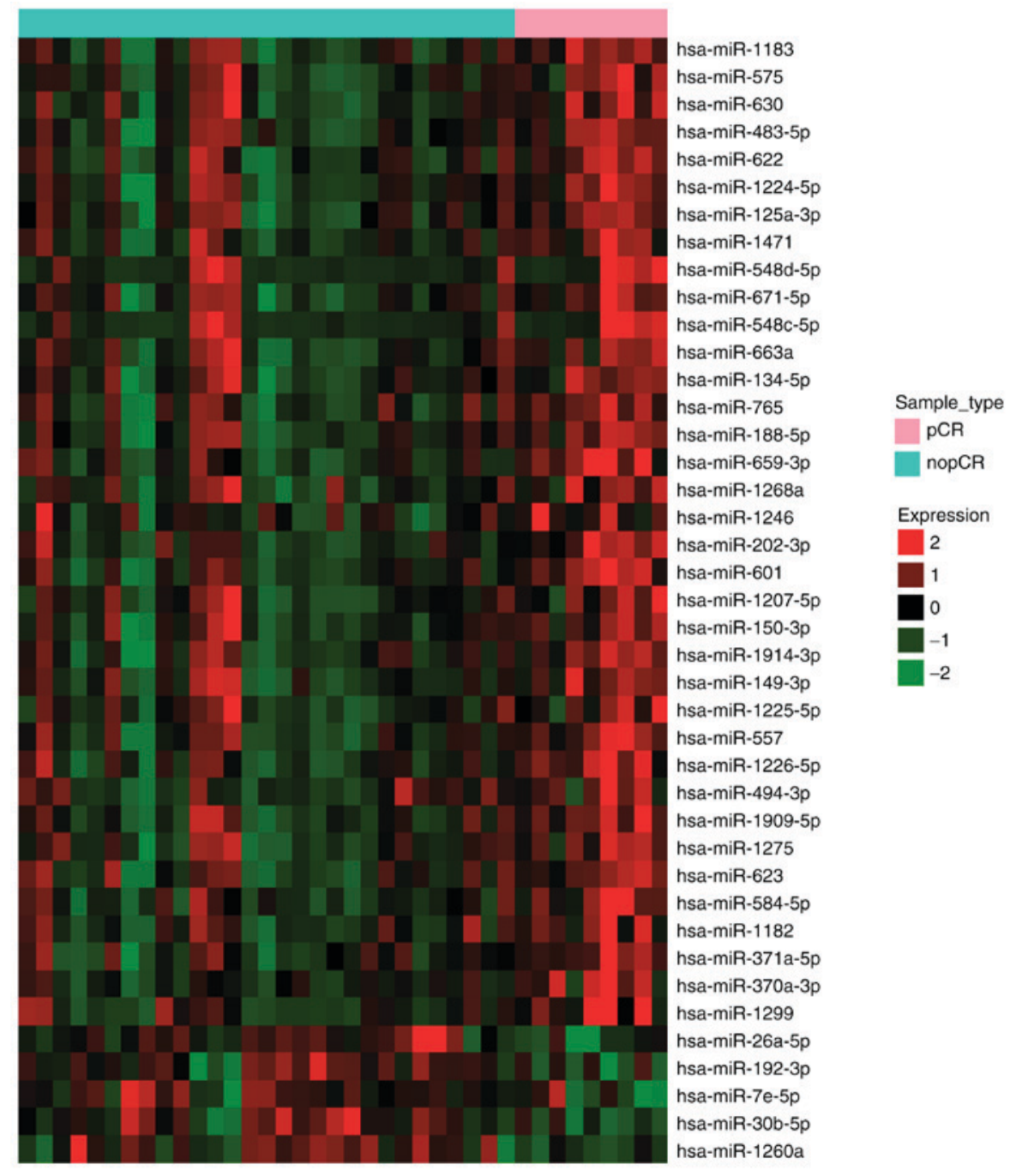

Figure 1. Heatmap for differentially expressed miRNAs. The pink bar represents the pCR group and the green bar represents the no pCR group. miR/miRNA, microRNA; nCRT, neoadjuvant chemoradiotherapy; pCR, complete response to nCRT; no pCR, incomplete response to nCRT.

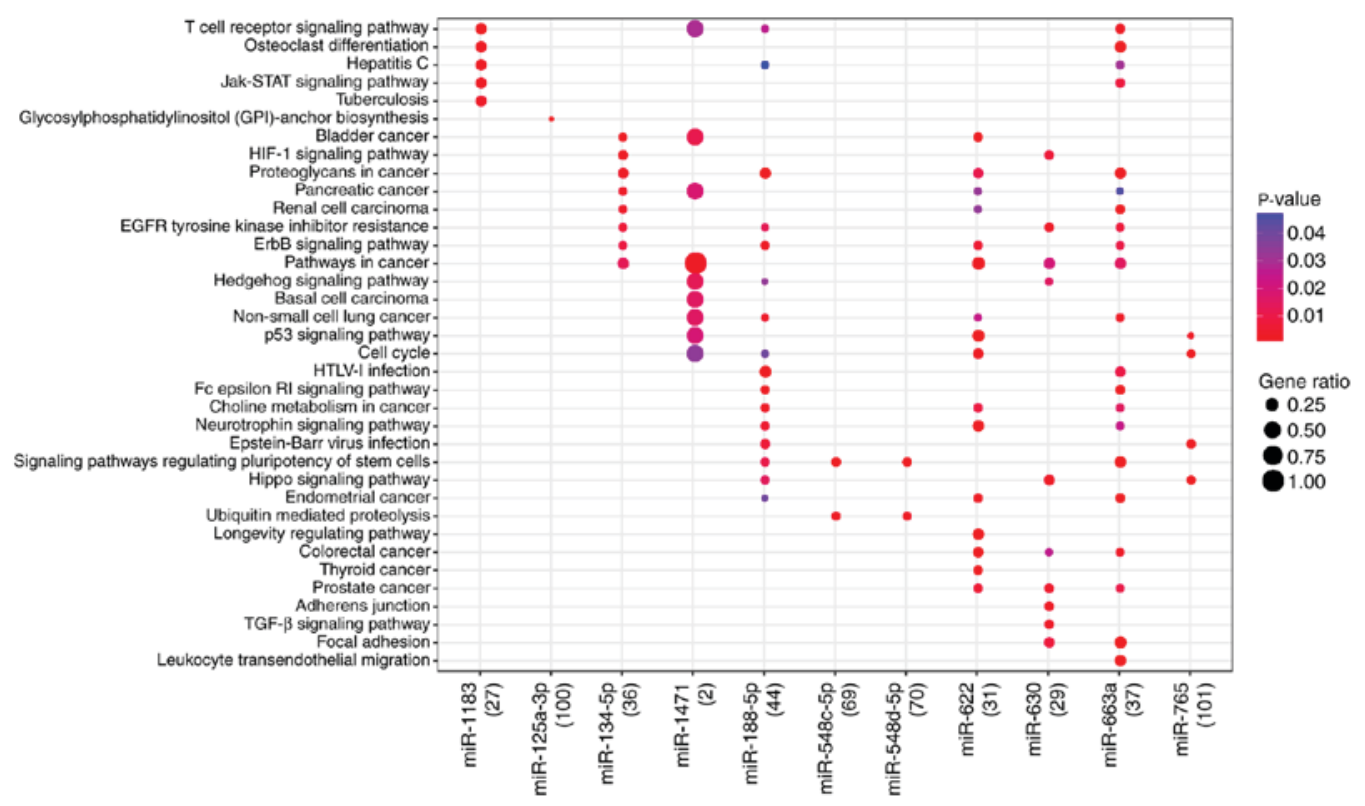

Figure 2. Enriched pathways for the top 11 differentially expressed miRNAs. Different dot color and dot size represent the P-value and gene ratio, respectively. The number below each miRNA name represents the number of target genes involved in pathway analysis. miR/miRNA, microRNA; STAT, signal transducer and activator of transcription; HIF, hypoxia inducible factor; EGFR, epidermal growth factor receptor; HTLV, human T-lymphotropic virus; TGF, transforming growth factor. 


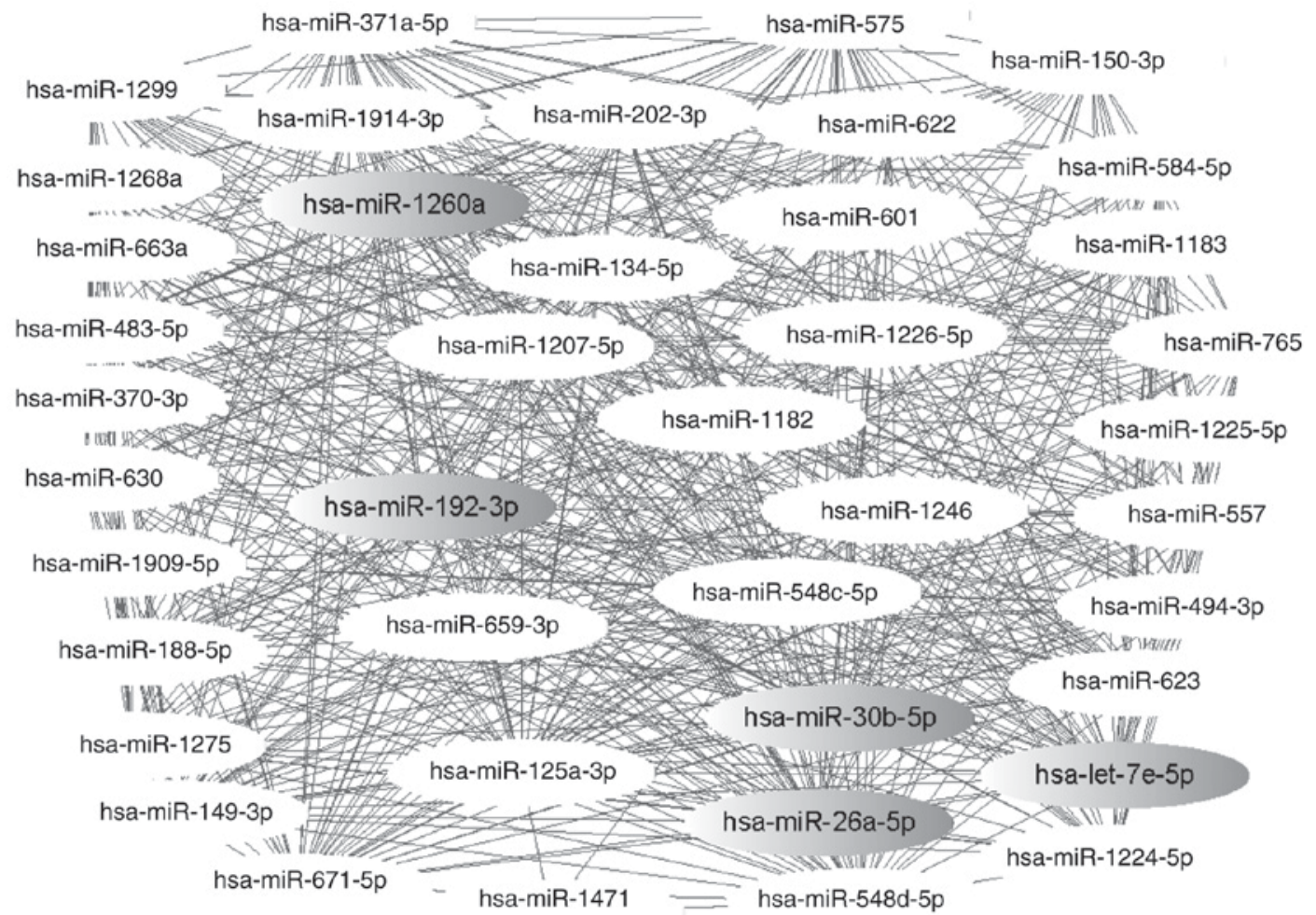

Figure 3. Co-regulatory network among miRNAs. Grey filled ovals represent downregulated miRNA and non-filled ovals represent upregulated miRNA in the pCR group compared with the no pCR group. miR/miRNA, microRNA; nCRT, neoadjuvant chemoradiotherapy; $\mathrm{pCR}$, complete response to nCRT; no pCR, incomplete response to nCRT.

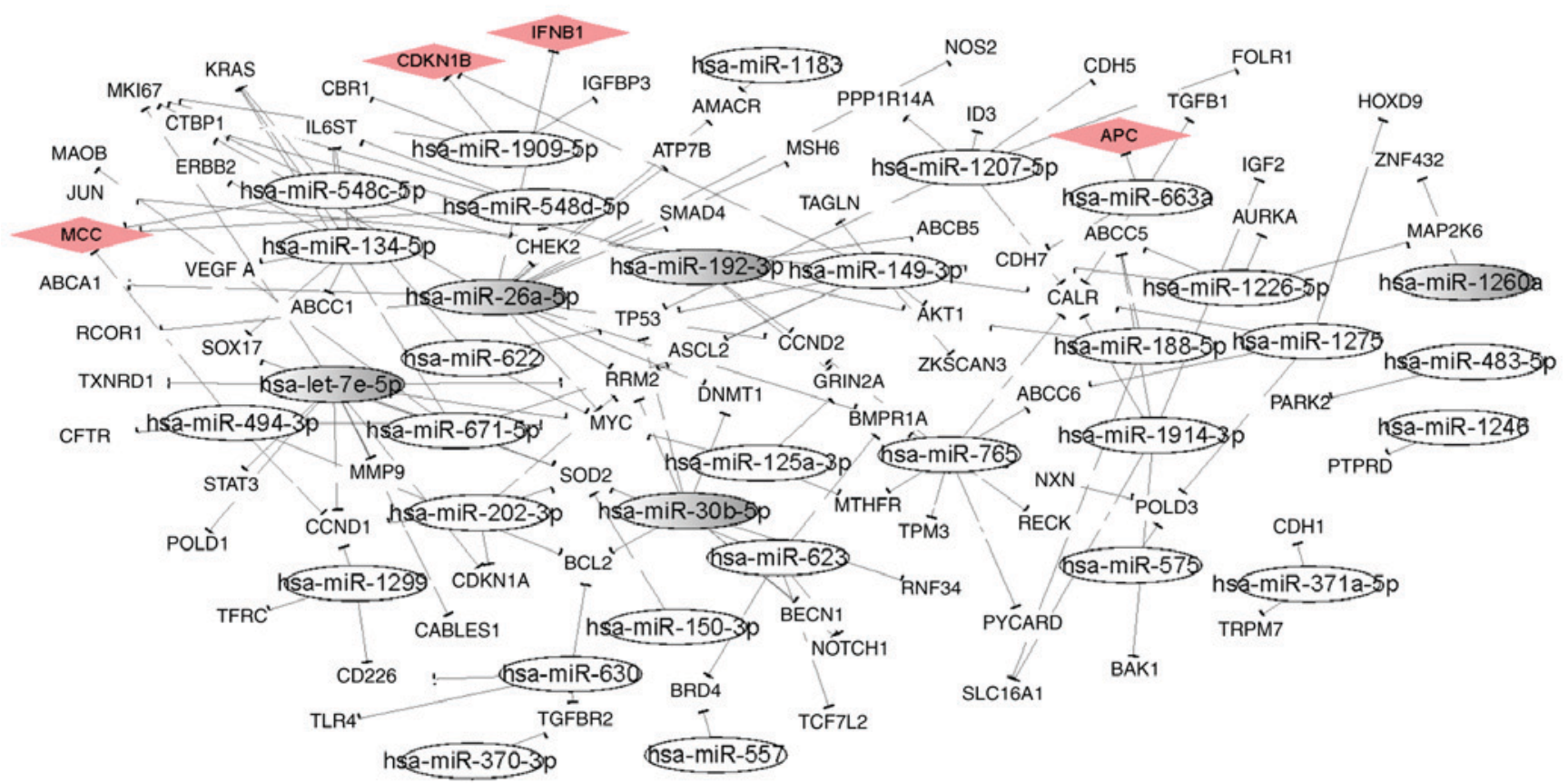

Figure 4. Regulatory network between miRNAs and target genes associated with colorectal neoplasms. Diamonds represent target genes, with pink diamonds representing genes associated with rectal cancer and all other genes being associated with colorectal neoplasms. Grey filled ovals represent downregulated miRNA and non-filled ovals represent upregulated miRNA in the pCR group compared with the no pCR group. miR/miRNA, microRNA; nCRT, neoadjuvant chemoradiotherapy; pCR, complete response to nCRT; no pCR, incomplete response to nCRT; MCC, mutated in colorectal cancers; CDKN1B, cyclin-dependent kinase inhibitor 1B; IFNB1, interferon $\beta 1$; APC, adenomatous polyposis coli; MKI67, marker of proliferation Ki-67; IL6ST, interleukin-6 signal transducer; CHEK2, cell cycle checkpoint kinase 2; CDH7, cadherin 7; CALR, calreticulin; TGFB1, transforming growth factor $\beta 1$.

the apoptosis, of liver cancer stem-like cells (28). In addition, miRNA-548d-5p was reported to have a complementary role in supporting oncogenicity in cervical cancer (29). However, few studies have reported the association between these two 
miRNAs and rectal cancer. Co-regulatory network analysis indicated that miR-548c-5p and miR-548d-5p may function as a complex to co-regulate four genes associated with colorectal neoplasms, including IL6ST, CHEK2, MKI67 and MCC. High IL-6 cytokine levels were previously reported to be associated with an advanced stage of disease and decreased survival of patients with CRC (30). A previous study demonstrated that IL6ST mediated tumor cell proliferation and apoptosis through glycoprotein 130 activation, with subsequent signaling through signal transducer and activator of transcription 3 in CRC (31). CHEK2 is a tumor suppressor that functions in the p53 pathway of the DNA damage response (32). The CHEK2 variants I157T and 1100 delC were reported to increase the risk of CRC $(33,34)$. MKI67 encodes a nuclear protein that is strictly associated with cellular proliferation, which means it is regarded as a proliferative marker (35). Antigen Ki-67, encoded by MKI67, is reported to be highly expressed in proliferative cells (36) and a previous study demonstrated that increased Ki-67 expression post-therapy was associated with improved disease-free survival in patients with rectal cancer (37). In addition, another study reported that the extent of the tumor response to nCRT was closely associated with the expression level of Ki-67 (38). Therefore, the expression of Ki-67 may be a potential biomarker for predicting the radiosensitivity of tumors for CRT in rectal cancers. A colorectal mutant cancer protein encoded by $M C C$, located on chromosome 5q21, was identified as a tumor suppressor gene and is considered to negatively regulate cell cycle progression (39). Allele loss in $M C C$ is common in CRC (40). These findings indicate that miR-548c-5p and miR-548d-5p may be involved in the mechanism of the CR to nCRT by targeting genes associated with cell proliferation, DNA damage and the cell cycle.

Furthermore, KEGG pathway analysis revealed that 'signaling pathways regulating pluripotency of stem cells' and 'ubiquitin-mediated proteolysis' signaling pathways were significantly enriched for the miR-548c-5p/miR-548d-5p complex. Cancer stem cells have the capacity for tumor initiation by differentiating into heterogeneous lineages of cancer cells (41). The ubiquitin-dependent proteolysis system is involved in the regulation $\beta$-catenin turnover (42) and it is established that the accumulation of $\beta$-catenin leads to the transcription of pre-oncogenes (43). These findings indicate that the miR-548c-5p/miR-548d-5p complex may contribute to the CR to nCRT through the pluripotency of stem cells and ubiquitin-mediated proteolysis pathways.

It was previously reported that miR-663 exhibited an anti-cancer effect by targeting the TGFB1 transcript through the TGF $\beta$ signaling pathway in SW480 human colon cancer cells (44), which is consistent with the results of the present study. In the present study, three other genes associated with colorectal neoplasms were demonstrated to be regulated by miR-663a, including $C D H 7, C A L R$ and $A P C$. Furthermore, E-cadherin (CDH1), a member of the $\mathrm{CDH}$ family, determines cell-cell adhesion and increases the nuclear translocation of $\beta$-catenin $(44,45)$. It is well-document that the APC protein, encoded by $A P C$, prevents the accumulation of $\beta$-catenin, a key factor in cancer (46). $\mathrm{CDH} 7$ is also a member of the CDH family (47). Thus, it can be suggested that miR-663a may function by altering the levels of $\beta$-catenin through targeting $\mathrm{CDH}$ and $A P C$. Calreticulin, encoded by $C A L R$, was reported to be associated with the infiltration of T-cells, and low expression of CALR may represent a novel mechanism underlying immune escape in colon cancer (48). Thus, miR-663a may be closely associated with a CR to nCRT by targeting these four genes.

The aforementioned results support the predictive roles of miR-548c-5p, miR-548d-5p and miR-663a in the response of patients with rectal cancer to nCRT. The present study further analyzed the miRNA microarray data developed from patients treated with capecitabine-oxaliplatin and pelvic conformal radiotherapy (45 cGy) followed by surgery (after 6-8 weeks). Due to the lack of the clinical samples under the same conditions, experimental validations were not available, which is a limitation of the present study. Thus, evaluations in larger, prospective trials are required to validate these biomarkers.

In conclusion, miR-548c-5p, miR-548d-5p and miR-663a are potential biomarkers for predicting a CR to $\mathrm{nCRT}$ in rectal cancer. The miR-548c-5p/miR-548d-5p complex may function by targeting IL6ST, CHEK2, MKI67 and MCC. In addition, this complex may function through the pluripotency of stem cells and ubiquitin-mediated proteolysis signaling pathways. miR-663a may affect the tumor response to treatment by regulating $C D H 7, C A L R, A P C$ and $T G F B 1$. Furthermore, targeting these miRNAs with oligonucleotides may represent a potential therapy for improving a poor response to nCRT in patients with rectal cancer.

\section{Acknowledgements}

Not applicable.

\section{Funding}

The present study was supported by the Bureau of Science and Technology of Innovation and Entrepreneurship Project (Lanzhou, China; grant no. 2015-RC-37).

\section{Availability of data and materials}

The datasets used and/or analyzed during the current study are available from the corresponding author on reasonable request.

\section{Authors' contributions}

$\mathrm{BD}$ and $\mathrm{WZ}$ were responsible for the conception and design of the present study. JW and LC acquired the data. XW and DW analyzed and interpreted the data. TW, XS and DW performed the statistical analyses. XY, DW and LC were involved in conception of the research, participated in its design and coordination and aided the writing of the manuscript. All authors read and approved the final manuscript.

\section{Ethics approval and consent to participate}

Not applicable.

\section{Consent for publication}

Not applicable. 


\section{Competing interests}

The authors declare that they have no competing interests.

\section{References}

1. Zheng S and Cai S: Colorectal cancer epidemiology and prevention study in China. Chinese-German J Clinical Oncol 2: 72-75, 2003.

2. Jemal A, Bray F, Center MM, Ferlay J, Ward E, and Forman D Global cancer statistics. CA Cancer J Clin 61: 69-90, 2011.

3. Jemal A, Siegel R, Ward E, Hao Y, Xu J, Murray T and Thun MJ: Cancer statistics, 2008. CA Cancer J Clin 58: 71-96, 2008.

4. Dusek L, Muzik J, Kubasek M, Koptikova J, Zaloudik J and Vyzula R: Epidemiology of Malignant Tumours in the Czech Republic. http://www.svod.cz. Accessed September 12, 2013.

5. Krook JE, Moertel CG, Gunderson LL, Wieand HS, Collins RT, Beart RW, Kubista TP, Poon MA, Meyers WC, Mailliard JA, et al Effective surgical adjuvant therapy for high-risk rectal carcinoma. N Engl J Med 324: 709-715, 1991.

6. Valentini V, Aristei C, Glimelius B, Minsky BD, Beets-Tan R, Borras JM, Haustermans K, Maingon P, Overgaard J, Pahlman L, et al: Multidisciplinary rectal cancer management: 2nd european rectal cancer consensus conference (EURECA-CC2). Radiother Oncol 92: 148-163, 2009.

7. Gérard JP, Conroy T, Bonnetain F, Bouché O, Chapet O, Closon-Dejardin MT, Untereiner M, Leduc B, Francois E, Maurel J, et al: Preoperative radiotherapy with or without concurrent fluorouracil and leucovorin in T3-4 rectal cancers: Results of FFCD 9203. J Clin Oncol 24: 4620-4625, 2006.

8. Engels B, Gevaert T, Sermeus A and Ridder MD: Current status of intensified neo-adjuvant systemic therapy in locally advanced rectal cancer. Front Oncol 2: 47, 2012.

9. Mandard AM, Dalibard F, Mandard JC, Marnay J, Henry-Amar M, Petiot JF, Roussel A, Jacob JH, Segol P, Samama G, et al: Pathologic assessment of tumor regression after preoperative chemoradiotherapy of esophageal carcinoma. Clinicopathologic correlations. Cancer 73: 2680-2686, 1994.

10. Martin ST, Heneghan HM and Winter DC: Systematic review and meta-analysis of outcomes following pathological complete response to neoadjuvant chemoradiotherapy for rectal cancer. $\mathrm{Br}$ J Surg 99: 918-928, 2012.

11. Suárez J, Vera R, Balén E, Gómez M, Arias F, Lera JM, Herrera J and Zazpe C: Pathologic response assessed by Mandard grade is a better prognostic factor than down staging for disease-free survival after preoperative radiochemotherapy for advanced rectal cancer. Colorectal Dis 10: 563-568, 2008.

12. Azizian A, Gruber J, Ghadimi BM and Gaedcke J: MicroRNA in rectal cancer. World J Gastrointest Oncol 8: 416-426, 2016.

13. Svoboda M, Sana J, Fabian P, Kocakova I, Gombosova J, Nekvindova J, Radova L, Vyzula R and Slaby O: MicroRNA expression profile associated with response to neoadjuvant chemoradiotherapy in locally advanced rectal cancer patients. Radiat Oncol 7: 195, 2012.

14. Drebber U,Lay M, Wedemeyer I, Vallböhmer D, Bollschweiler E, Brabender J, Mönig SP, Hölscher AH, Dienes HP and Odenthal M: Altered levels of the onco-microRNA 21 and the tumor-supressor microRNAs 143 and 145 in advanced recta cancer indicate successful neoadjuvant chemoradiotherapy. Int J Oncol 39: 409-415, 2011

15. Kheirelseid EA, Miller N, Chang KH, Curran C, Hennessey E, Sheehan M, Newell J, Lemetre C, Balls G and Kerin MJ: miRNA expressions in rectal cancer as predictors of response to neoadjuvant chemoradiation therapy. Int J Colorectal Dis 28: 247-260, 2013.

16. Lopes-Ramos CM, Habr-Gama A, Quevedo Bde S, Felício NM, Bettoni F, Koyama FC, Asprino PF, Galante PA, Gama-Rodrigues J, Camargo AA, et al: Overexpression of miR-21-5p as a predictive marker for complete tumor regression to neoadjuvant chemoradiotherapy in rectal cancer patients. BMC Med Genomics 7: 68, 2014.

17. Della Vittoria Scarpati G, Falcetta F, Carlomagno C, Ubezio P, Marchini S, De Stefano A, Singh VK, D'Incalci M, De Placido S and Pepe S: A Specific miRNA signature correlates with complete pathological response to neoadjuvant chemoradiotherapy in locally advanced rectal cancer. Int J Radiat Oncol Biol Phys 83: 1113-1119, 2012
18. Smyth BGK: Limma: Linear models for microarray data. In Bioinformatics and Computational Biology Solutions Using R and Bioconductor. Springer, p397-420, 2005.

19. Griffiths-Jones S: miRBase: The microRNA sequence database. Methods Mol Biol 342: 129-138, 2006.

20. Griffiths-Jones S: miRBase: microRNA sequences and annotation. Curr Protoc Bioinformatics 29: 12.9.1-12.9.10, 2010.

21. Dweep H and Gretz N: miRWalk2.0: A comprehensive atlas of microRNA-target interactions. Nat Methods 12: 697, 2015.

22. Yu G, Wang LG, Han Y and He QY: clusterProfiler: An R package for comparing biological themes among gene clusters. OMICS 16: 284-287, 2012.

23. Maqbool A, Lattke M, Wirth T and Baumann B: Sustained, neuron-specific IKK/NF- $\kappa \mathrm{B}$ activation generates a selective neuroinflammatory response promoting local neurodegeneration with aging. Mol Neurodegener 8: 40, 2013.

24. Kohl M, Wiese S and Warscheid B: Cytoscape: Software for visualization and analysis of biological networks. Methods Mol Biol 696: 291-303, 2011

25. Davis AP, Grondin CJ, Lennonhopkins K, Saraceni-Richards C, Sciaky D, King BL, Wiegers TC and Mattingly CJ: The Comparative Toxicogenomics Database's 10th year anniversary: Update 2015. Nucleic Acids Res 43 (Database issue): D914-D920, 2015.

26. Semenza GL: HIF-1 and mechanisms of hypoxia sensing. Curr Opin Cell Biol 13: 167-171, 2001.

27. Jaakkola P, Mole DR, Tian YM, Wilson MI, Gielbert J, Gaskell SJ, von Kriegsheim A, Hebestreit HF, Mukherji M, Schofield CJ, et al: Targeting of HIF-alpha to the von Hippel-Lindau ubiquitylation complex by O2-regulated prolyl hydroxylation. Science 292: 468-472, 2001.

28. Fang L, Zhang HB, Li H, Fu Y and Yang GS: miR-548c-5p inhibits proliferation and migration and promotes apoptosis in CD90(+) HepG2 cells. Radiol Oncol 46: 233-241, 2015.

29. Mandal P, Bhattacharjee B, Das Ghosh D, Mondal NR, Roy Chowdhury R, Roy S and Sengupta S: Differential expression of HPV16 L2 gene in cervical cancers harboring episomal HPV16 genomes: Influence of synonymous and non-coding region variations. PLoS One 8: e65647, 2013.

30. Knüpfer $\mathrm{H}$ and Preiss R: Serum interleukin-6 levels in colorectal cancer patients-a summary of published results. Int J Colorectal Dis 25: 135-140, 2010.

31. Waldner MJ, Foersch S and Neurath MF: Interleukin-6-A Key regulator of colorectal cancer development. Int J Biol Sci 8: 1248-1253, 2012.

32. Kilpivaara O, Laiho P, Aaltonen LA and Nevanlinna $\mathrm{H}$ : CHEK2 1100delC and colorectal cancer. J Med Genet 40: e110, 2003

33. Meijers-Heijboer H, Wijnen J, Vasen H, Wasielewski M, Wagner A, Hollestelle A, Elstrodt F, van den Bos R, de Snoo A, Fat GT, et al: The CHEK2 1100delC mutation identifies families with a hereditary breast and colorectal cancer phenotype. Am J Hum Genet 72: 1308-1314, 2003.

34. Kilpivaara O, Alhopuro P, Vahteristo P, Aaltonen LA and Nevanlinna H: CHEK2 I157T associates with familial and sporadic colorectal cancer. J Med Genet 43: e34, 2006.

35. Hofstädter F, Knüchel R and Rüschoff J: Cell proliferation assessment in oncology. Virchows Arch 427: 323-341, 1995.

36. Dalerba P, Kalisky T, Sahoo D, Rajendran PS, Rothenberg ME, Leyrat AA, Sim S, Okamoto J, Johnston DM, Qian D, et al: Single-cell dissection of transcriptional heterogeneity in human colon tumors. Nat Biotechnol 29: 1120-1127, 2011.

37. Rau B, Sturm I, Lage H, Berger S, Schneider U, Hauptmann S, Wust P, Riess H, Schlag PM, Dörken B and Daniel PT: Dynamic Expression Profile of p21WAF1/CIP1 and Ki-67 predicts survival in rectal carcinoma treated with preoperative radiochemotherapy. J Clin Onco 21: 3391-3401, 2003.

38. Kim NK, Park JK, Lee KY, Yang WI, Yun SH, Sung J and Min JS: p53, BCL-2, and Ki-67 expression according to tumor response after concurrent chemoradiotherapy for advanced rectal cancer. Ann Surg Oncol 8: 418-424, 2001.

39. Kinzler KW, Nilbert MC, Vogelstein B, Bryan TM, Levy DB, Smith KJ, Preisinger AC, Hamilton SR, Hedge P, Markham A, et al: Identification of a gene located at chromosome $5 \mathrm{q} 21$ that is mutated in colorectal cancers. Science 251 1366-1370, 1991.

40. Cawkwell L, Lewis FA, and Quirke P: Frequency of allele loss of DCC, p53, RBI, WT1, NF1, NM23 and APC/MCC in colorectal cancer assayed by fluorescent multiplex polymerase chain reaction. Br J Cancer 70: 813-818, 1994 
41. Kong D, Li Y, Wang Z and Sarkar FH: Cancer stem cells and epithelial-to-mesenchymal transition (EMT)-phenotypic cells: Are they cousins or twins? Cancers (Basel) 3: 716-729, 2011.

42. Aberle H, Bauer A, Stappert J, Kispert A and Kemler R: beta-catenin is a target for the ubiquitin- proteasome pathway. EMBO J 16: 3797-3804, 1997.

43. Morin PJ, Sparks AB, Korinek V, Barker N, Clevers H, Vogelstein B and Kinzler KW: Activation of beta-catenin-Tcf signaling in colon cancer by mutations in beta-catenin or APC. Science 275: 1787-1790, 1997.

44. Zhu L, Chen H, Zhou D, Li D, Bai R, Zheng S and Ge W: MicroRNA-9 up-regulation is involved in colorectal cancer metastasis via promoting cell motility. Med Oncol 29: 1037-1043, 2012.

45. Schmalhofer O, Brabletz S and Brabletz T: E-cadherin, beta-catenin, and ZEB1 in malignant progression of cancer. Cancer Metastasis Rev 28: 151-166, 2009.
46. Munemitsu S, Albert I, Souza B, Rubinfeld B and Polakis P: Regulation of intracellular beta-catenin levels by the adenomatous polyposis coli (APC) tumor-suppressor protein. Proc Natl Acad Sci USA 92: 3046-3050, 1995.

47. Gul IS, Hulpiau P, Saeys Y and Van RF: Evolution and diversity of cadherins and catenins. Exp Cell Res 358: 3-9, 2017.

48. Peng RQ, Chen YB, Ding Y, Zhang R, Zhang X, Yu XJ, Zhou ZW, Zeng YX and Zhang XS: Expression of calreticulin is associated with infiltration of T-cells in stage IIIB colon cancer. World J Gastroenterol 16: 2428-2434, 2010.

This work is licensed under a Creative Commons Attribution-NonCommercial-NoDerivatives 4.0 International (CC BY-NC-ND 4.0) License. 